Article

\title{
Structural Elucidation of the DFG-Asp in and DFG-Asp out States of TAM Kinases and Insight into the Selectivity of Their Inhibitors
}

\author{
Abdellah Messoussi ${ }^{1,2}$, Lucile Peyronnet ${ }^{1}$, Clémence Feneyrolles ${ }^{1}$, Gwénaël Chevé ${ }^{1}$, \\ Khalid Bougrin ${ }^{2}$ and Aziz Yasri ${ }^{1, *}$
}

1 OriBase Pharma, Parc Euromedecine, Cap Gamma, 1682, rue de la Valsière, 34189 Montpellier, France; E-Mails: amessoussi@oribase-pharma.com (A.M.);

lpeyronnet@oribase-pharma.com (L.P.); cfeneyrolles@oribase-pharma.com (C.F.); gcheve@oribase-pharma.com (G.C.)

2 Laboratoire de Chimie des Plantes et de Synthèse Organique et Bioorganique, URAC23, Université Mohammed V, Faculté des Sciences B.P., 1014 Rabat, Morocco; E-Mail: khalid.bougrin@smct-ma.com

* Author to whom correspondence should be addressed; E-Mail: ayasri@oribase-pharma.com; Tel.: +33-04-67-727-670; Fax: +33-04-67-727-679.

External Editor: Philippe Belmont

Received: 31 July 2014; in revised form: 24 September 2014 / Accepted: 26 September 2014 / Published: 10 October 2014

\begin{abstract}
Structural elucidation of the active (DFG-Asp in) and inactive (DFG-Asp out) states of the TAM family of receptor tyrosine kinases is required for future development of TAM inhibitors as drugs. Herein we report a computational study on each of the three TAM members Tyro-3, Axl and Mer. DFG-Asp in and DFG-Asp out homology models of each one were built based on the X-ray structure of c-Met kinase, an enzyme with a closely related sequence. Structural validation and in silico screening enabled identification of critical amino acids for ligand binding within the active site of each DFG-Asp in and DFG-Asp out model. The position and nature of amino acids that differ among Tyro-3, Axl and Mer, and the potential role of these residues in the design of selective TAM ligands, are discussed.
\end{abstract}

Keywords: tyrosine kinase; TAM kinase family; homology model; kinase selectivity 


\section{Introduction}

\subsection{Kinases as Therapeutic Targets}

Kinases are ubiquitous enzymes from the transferase family. Protein kinases catalyze the covalent transfer of a phosphoryl group from ATP to a substrate, which can be another protein (e.g., another kinase), a lipid or a nucleic acid. Protein kinases are the largest family of kinases, encompassing 518 members (as currently identified in the human genome) [1]. Their catalytic domain is well conserved and features a common set of crucial amino acids. All protein kinases possess an $\mathrm{Mg}^{2+}$-ATP binding site, a protein-substrate binding site and multiple regulator sites. One interesting class of protein kinases are receptor tyrosine kinases (RTKs), transmembrane proteins that transmit signals from the extracellular medium to the cytoplasm and nucleus. They are fundamental for basic life functions, as they regulate many cellular processes such as survival, growth, differentiation, adhesion and motility. Dysfunction or deregulation of certain kinases can lead to diseases such as cancer; thus, these enzymes have garnered enormous interest as therapeutic targets.

\subsection{The TAM Family as Therapeutic Targets}

The TAM family of protein RTKs was named after its three members: Tyro-3 (also called Sky), Axl (Ark or Ufo), and Mer (MerTK). TAM kinases contain three structural domains: an extracellular domain, which binds the natural ligand and comprises two immunoglobulin-like and two fibronectin domains [2]; an intracellular domain, which contains the tyrosine kinase domain and exhibits a well conserved sequence that defines the TAM family (KW-(I/L)-A-(I/L)-ES) [3]; and a transmembrane domain, which links the other two domains.

TAM kinases were initially found in cancer cells [4-7], which lead to the discovery that overexpression or ectopia of these RTKs contribute to carcinogenesis [8-10]. Indeed, many cancers, including cancers of the uterine endometrium [8], stomach [11,12], colon [13], prostate [14-17], thyroid [18-20], lung [21,22], breast [23-26], ovaries [27,28], liver [29] and kidneys [9], as well as glioblastomas [30], melanomas [31-33], osteosarcomas [34], leukemias [4,35,36] and multiple myelomas [37] exhibit altered expression of one or more TAM members [3]. Interestingly, among the three TAM members, Axl has been found to be the most involved in human cancers [3]. Moreover, in cancer Axl is implicated in drug-resistance mechanisms [38-41] and associated with poor prognosis and a high level of recurrence [42]. All of these findings corroborate the idea that TAM kinases are attractive potential targets for oncology treatment.

\subsection{Structure-Based Drug Design for Lead Identification and Optimization}

Over the past decade, identification of new drug targets has been greatly facilitated by the combination of classical biochemistry tools with new technologies such as proteomics. Furthermore, advances in analytical techniques such as NMR (higher frequency and technology) and crystallography (HTS, synchrotron radiation, phase resolution, etc.) have radically shortened the time needed to solve the structure of potential therapeutic targets. This progress has renewed interest in rational medicinal chemistry through Structure-Based Drug Design (SBDD), an approach that has gradually brought new 
drugs to market, including Viracept ${ }^{\circledR}$ (nelfinavir; Agouron) $[43,44]$ and Agenerase ${ }^{\circledR}$ (amprenavir; Vertex and GlaxoSmithKline) [45].

\subsection{Comprehension of Binding-Mode for the Design of Specific Enzyme Inhibitors}

Understanding binding modes is especially important for kinases, given the high conservation of their catalytic domain, to which classical ATP competitive inhibitors (type I and II) tend to bind. Selectivity stems from interactions of the inhibitor with less conserved parts of the kinase domain. However, designing a synthetic inhibitor that can reach these selectivity pockets is not trivial: in the absence of a 3D structure of the target, ligand design requires intense Structure-Activity-Relationship (SAR) analyses and exhaustive chemical synthesis.

The first step in SBDD studies is structural elucidation of the target, which can be done by X-ray crystallography or NMR. The next step is to assess the binding behavior between protein and ligand. If no data are available in the Protein Data Bank (PDB), then homology modeling can be used to this end.

Presently, there are no potent selective inhibitors of any TAM kinase on the market [46]. Given the widespread expression of these enzymes (Tyro-3 is found mainly in the central nervous system; Axl is ubiquitous; and Mer is found chiefly in macrophages and NK cells [3]), inhibitors of any single TAM must be highly selective. For kinase inhibitor drugs, selectivity is not merely a question of efficacy, it is also a requisite for safety. However, the lack of selectivity is tolerated in some therapeutical indications such as cancer.

In the work described here, we sought to study the activity domain of each of the three TAM kinases (Tyro-3, Axl and Mer) in each of their two conformations (DFG-Asp in and DFG-Asp out). Hence, we designed and validated relevant homology models, and then studied their active sites. We performed virtual screening of TAM inhibitors against these models, gaining insight into inhibitor/kinase selectivity and invaluable knowledge for the future design of scaffolds for new, active and selective TAM inhibitors.

\section{Results and Discussion}

\subsection{Homology Modeling of the TAM Family}

None of the TAM kinase 3D structures was solved in the DFG-Asp out conformation; thus, they were built by homology modeling using as template the phylogenetically-related tyrosine kinase c-Met in this conformation (PDB ID: 3F82 [47]). In fact, the identity percentages between each of the three TAM kinases and c-Met are above 45\%: the values are $45.42 \%$ for Tyro-3, 45.98\% for Axl and 45.04\% for Mer.

Crystal structures of Mer and Tyro-3 in the DFG-Asp in conformation were published in 2009 (PDB ID: 2P0C, 3BRB, 3BPR [48]), 2012 (PDB ID: 3TCP, 3QUP) [49,50] and 2013 (PDB ID: 4M3Q, 4MH7, 4MHA, 4FEQ, 4FF8 [51]). However, all these 3D structures correspond to murine proteins in their DFG-Asp in state and lack a portion of the activation loop. Consequently, as we wanted to study the whole kinase domain with its activation loop for the human kinases, we decided to build 3D models for the three kinases in the DGF-Asp in state using X-ray structure of c-Met kinase as a template. This 3D structure corresponds to the human c-Met kinase and it has the activation loop completely characterized (PDB ID: 2WD1 [52])). 


\subsection{Validation of the TAM Kinase Models}

A total of six models was built (three TAM kinases x two states), and then structurally validated by checking the torsion angles for each amino acid. These calculations were performed using Procheck software, which generates Ramachandran plots. The three DFG-Asp out models possess $88.4 \%$ (Tyro-3), $87.9 \%(\mathrm{Axl})$ and $86.8 \%$ (Mer) of the amino acids in the favorable regions; and the three DFG-Asp in models, 90.8\% (Tyro-3), 88.8\% (Axl) and 87.7\% (Mer) (Supplementary Figure S1). The amino acids outside of the favorable region are located on the protein surface, which is exposed to the solvent and is not subjected to the docking process. Since the DFG-Asp in crystal structures of Mer and of Tyro-3 in the literature are incomplete, we further validated our DFG-Asp in models of these two TAM kinases by superimposing them over the corresponding reported structures (Figures 1 and 2).

Figure 1. Alignment of all Mer structures from PDB with our Mer DFG-Asp in model. PDB structure of Mer in different shades of red (PDB ID 2P0C in dark salmon; PDB ID 3BPR in TV red; PDB ID 3BRB in firebrick; PDB ID 3TCP in raspberry; PDB ID 4M3Q in orange, PDB ID 4MH7 in bright orange; PDB ID 4MHA in red); our Mer DFG-Asp in model, in yellow (all colors from PyMOL). (a) Superimposition of reported Mer crystal structures with our Mer DFG-Asp in model. (b) Zoom on the activation loop (circled in white) found only in our model.
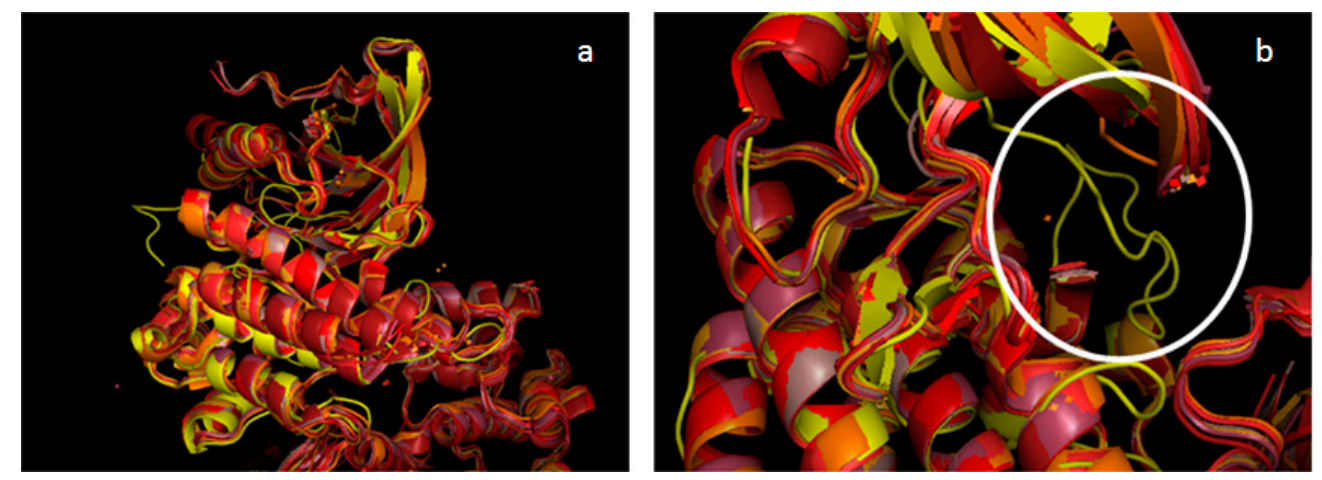

Figure 2. Alignment of all Tyro-3 structures from PDB with our Tyro-3 DFG-Asp in model. PDB structure of Tyro-3 in different shades of red (PDB ID 3QUP in TV red; PDB ID 4FF8 in firebrick; PDB ID 4FEQ in raspberry); our Tyro-3 DFG-Asp in model, in slate blue (all colors from PyMOL). (a) Superimposition of Tyro-3 crystal structures with our Tyro-3 DFG-Asp in model. (b) Zoom on the activation loop (circled in white) found only in our model.
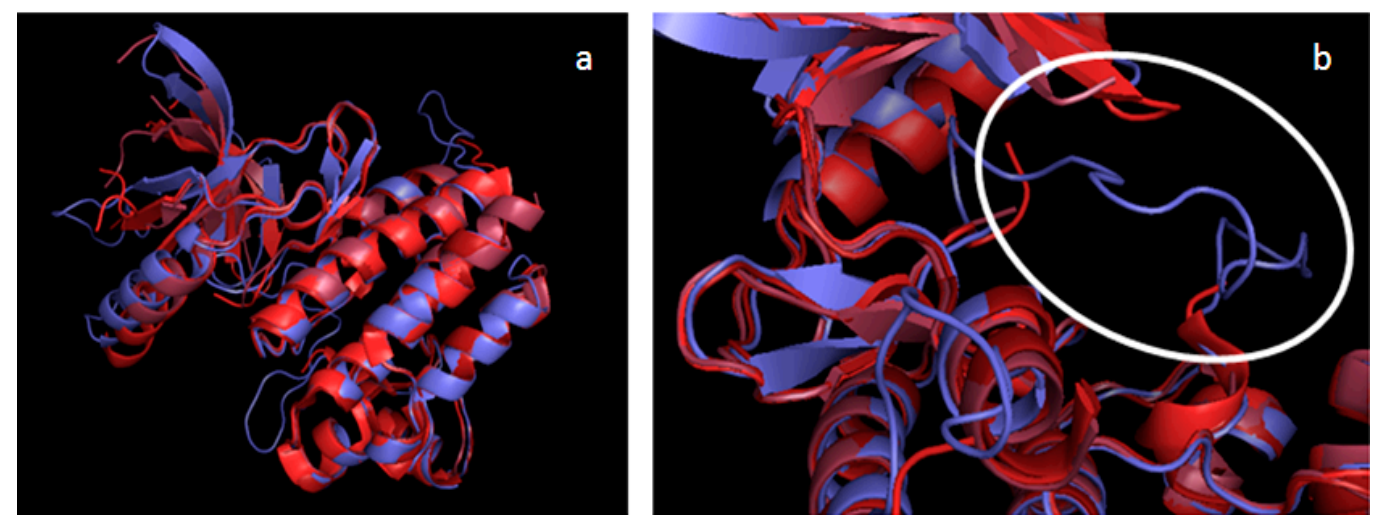
We also validated each of our six models, by docking a known efficient inhibitor into each one. For the DFG-Asp out models, the docking ligand used was BMS-777607, as it has previously been crystallized with the template kinase, $\mathrm{c}-\mathrm{Met}\left(\mathrm{IC}_{50}=3.9 \mathrm{nM}\right)$, and is active against all three of the TAM kinases (from highest to lowest activity: IC50 Axl = $1.1 \mathrm{nM}$; IC50 Tyro-3 = $4.3 \mathrm{nM}$; and $\mathrm{IC}_{50} \mathrm{Mer}=14 \mathrm{nM}$ ).

The position of BMS-777607 in the active site of three TAM kinases in the DFG-Asp out state is very closely related to its crystallized position in c-Met (PDB ID: 3F82) (Figure 3). Thus, the three DFG-Asp out models were validate again by a different method.

Figure 3. In silico screening of the DFG-Asp out homology models, using BMS777607. Tyro-3 in purple; Axl in green; Mer in yellow; and BMS777607 from the crystal structure of c-Met (PDB ID:3F82), in white. In each case, the color of BMS777607 is the same as that of the docking target.

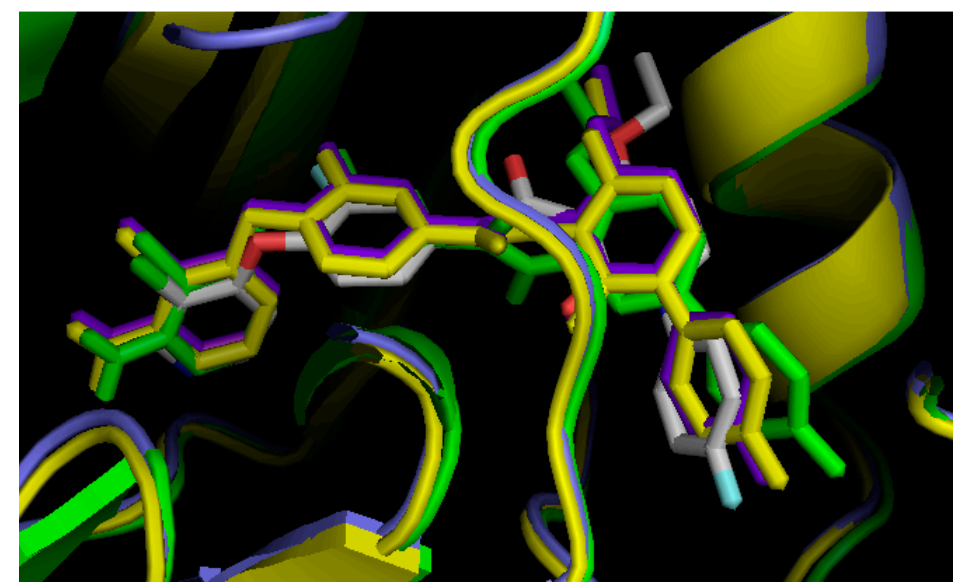

For the DFG-Asp in models of Tyro-3 and of Axl, the docking ligand used was UNC569, as it has already been crystallized with Mer (PDB ID: 3TCP; $\mathrm{IC}_{50}=2.9 \mathrm{nM}$ ) and inhibits both of these TAM kinases ( $\mathrm{IC}_{50}$ Tyro-3 $=48 \mathrm{nM}$; and $\mathrm{IC}_{50} \mathrm{Axl}=37 \mathrm{nM}$ ) [53]. The position of UNC569 in each of the three TAM kinases is close to that in the crystal structure of Mer (Figure 4). Hence, the three DFG-Asp in models were validated another time.

Figure 4. In silico screening of the DFG-Asp in homology models, using UNC569. Tyro-3 in purple, Axl in green; Mer in yellow; and UNC569 from the crystal structure of Mer (PDB ID: 3TCP), in white. In each case, the color of UNC569 is the same as that of the docking target.

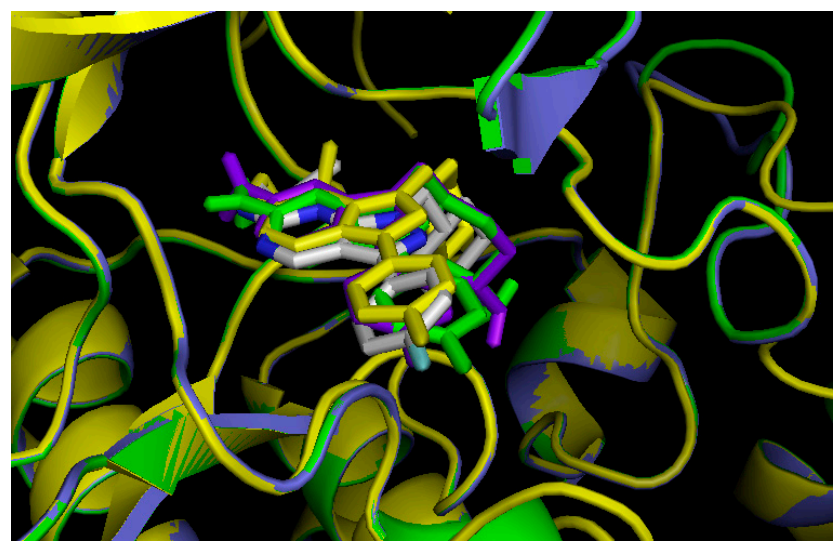




\title{
2.3. Comparison of Active Sites
}

To account for the variability in the active sites among the three TAM kinases in each conformation, we delimited volumes at $4 \AA, 5 \AA$ and $6 \AA$ from any atom of the docked ligands, using PyMol software.

The ligands used for delimiting these volumes were BMS-777607 (crystallized with C-Met in its DFG-Asp out state; PDB ID: 3F82) for the DFG-Asp out state, and UNC569 (crystallized with Mer in its DFG-Asp in state; PDB ID: 3TCP) for the DFG-Asp in state. We then enumerated all the amino acid sequences contained within these volumes. As representative examples, we show here the set of amino acids encompassed at $6 \AA$ for each TAM kinase model in the DFG-Asp in (Figure 5a) and the DFG-Asp out (Figure 5b) conformations.

Figure 5. Alignment of the three TAM kinase domains. The active site is highlighted at $6 \AA$ from the docked ligands. Identical residues are shown in green; non-conserved residues; in blue; and the key residue for each kinase, in orange. (a) Sequence alignment for the DFG-Asp in conformation. (b) Sequence alignment for the DFG-Asp out conformation.

\begin{abstract}
SP |sp|Q06418|518-790|TYRO3_HUMAN FTLGRMLGKGEFGSVREAQLKQEDGSFVKVAVKMLKADI IASSDIEEFLREAACMKEFDH 577 SP Isp|Q12866|587-858|MERTK_HUMAN LILGKILGEGEFGSVMEGNLKQEDGTSLKVAVKTMKLDNSSQREIEEFLSEAACMKDFSH 646 SP|sp|P30530|536-807|UFO_HUMMAN VALGKTLGEGEFGAVMEGQLNQDD-SILKVAVKTMKIAICTRSELEDFLSEAVCMKEFDH 594

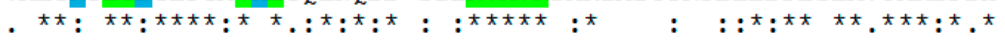

SP |sp |Q06418|518-790|TYRO3_HUMAN PHVAKLVGVSLRSRAKGRLPIPMVILPFMKHGDLHAFLLÄSRIGENPFNLPLQTLIRFMV 637 SP|sp|Q12866 | 587-858|MERTK_HUMAN PNVIRLLGVCIEMSS-QGIPKPMVILPFMKYGDLHTYLLYSRLETGPKHIPLQTLLKFMV 705 SP | sp | P30530| 536-807|UFO_HŪMAN PNVMRLIGVCFQGSERESFPAPVVILPFMKHGDLHSFLLYSRLGDQPVYLPTQMLVKFMA 654

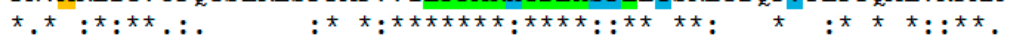

SP|sp | Q06418|518-790|TYRO3_HUMAN DIACGMEYLSSRNF IHRDLAARNCMIAEDMTVCVADFGLSRKIYSGDYYRQGCASKLPVK 697 SP|sp|Q12866|587-858|MERTK_HUMAN DIALGMEYLSNRNFLHRDLAARNCMIRDDMTVCVADFGLSKKIYSGDYYRQGRIAKMPVK 765 SP I SP | P30530|536-807|UFO_HÜMAN DIASGMEYLSTKRF IHRDLAARNCMLNENMSVCVADFGLSKKIYNGDYYRQGRIAKMPVK 714

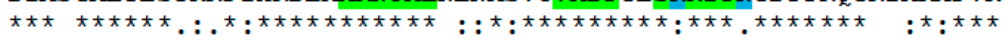

SP |sp|Q06418 | 518-790|TYRO3 HUMAN WLALESLADNLYTVQSDVWAFGVTMWEIMTRGQT PYAGIENAEI YNYLIGGNRLKQPPEC 757 SP | Sp |Q12866 | 587-858|MERTK_HUMAN WIAIESLADRVYTSKSDVWAFGVTMWEIATRGMT PYPGVQNHEMYDYLLHGHRLKQPEDC 825 SP | sp | P30530 | 536-807 |UFO_HŪMAN WIAIESLADRVYTSKSDVWSFGVTMWEIATRGQT PYPGVENSEIYDYLRQGNRLKQPADC 774

SP|Sp |Q06418|518-790|TYRO3_HUMAN MEDVYDLMYQCWSADPKQRPSFTCLRMELENIL 790

SP|sp|Q12866|587-858|MERTK_HUMAN LDELYEIMYSCWRTDPLDRPTFSVLRLQLEKLL 858

SP|sp|P30530|536-807|UFO_HUMAN LDGLYALMSRCWELNPQDRPSFTELREDLENTL 807

(a)

SP |sp |Q06418|518-790|TYRO3_HUMAN FTLGRMLGKGEFGSVRFAQLKQEDGSFVKVAVKMLKADI IASSDIEEFLREAACMKEFDH 577 SP|sp|Q12866|587-858|MERTK HUMAN LILGKILGEGEFGSVMEGNLKQEDGTSLKVAVKTMKLDNSSQRE IEEFLSEAACMKDFSH 646 SPIsp | P30530|536-807|UFO_HŪMAN VALGKTLGEGEFGAVMEGQLNQDD-SILKVAVKTMKIAICTRSELEDFLSEAVCMKEEDH 594

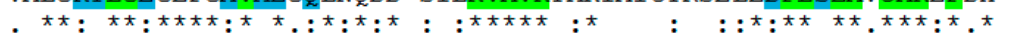

SP |sp|Q06418 | 518-790|TYRO3_HUMAN PHVAKLVGVSLRSRAKGRLPIPMVILPFMKHGDLHAFLLASRIGENPFNLPLQTLIRFMV 637 SP|sp|Q12866|587-858|MERTK_HUMAN PNVIRLLGVCIEMSS-QGIPKPMVILPFMKYGDLHTYLLYSRLETGPKHIPLQTLLKFMV 705 SP|sp|P30530|536-807|UFO_HŪMAN PNVMRLIGVCFQGSERESFPAPVVILPFMKHGDLHSFLLYSRLGDQPVYLPTQMLVKFMA 654

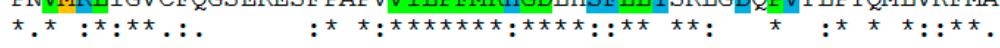

SP | sp | Q06418|518-790|TYRO3_HUMAN DIACGMEYLSSRNFIHRDLAARNCMLAEDMTVCVADFGLSRKIYSGDYYRQGCASKLPVK 697 SP | Sp | Q12866 | 587-858| MERTK_HUMAN DIALGMEYLSNRNELHRDLAARNCMLRDDMTVCVADFGLSKKIYSGDYYRQGRIAKMPVK 765 SP | sp | P30530| 536-807|UFO_HUMAN DIASGMEYLSTKRF IHRDLAARNCMLNENMSVCVADFGLSKKKIYNGDYYRQGRIAKMPVK 714

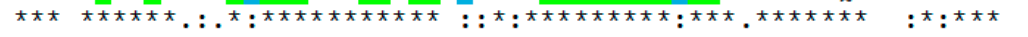

SP|sp|Q06418|518-790|TYRO3 HUMAN WLALESLADNLYTVQSDVWAFGVTMWEIMTRGQTPYAGIENAEIYNYLIGGNRLKQPPEC 757 SP|SP|Q12866 |587-858|MERTK_HUMAN WIAIESLADRVYTSKSDVWAFGVTMWEIATRGMT PYPGVQNHEMYDYLLHGHRLKQPEDC 825 SP | sp | P30530 | 536-807| UFO_HUMMAN WIAIESLADRVYTSKSDVWSFGVTMWEIATRGQTPYPGVENSEIYDYLRQGNRLKQPADC 774

SP|SP|Q06418|518-790|TYRO3 HUMAN MEDVYDLMYQCWSADPKQRPSFTCLRMELENIL 790 SP|SP|Q12866|587-858|MERTK_HUMAN LDELYEIMYSCWRTDPLDRPTFSVLRLQLEKLL 858 SP |sp | P30530|536-807|UFO_HUMAN LDGLYALMSRCWELNPQDRPSFTELREDLENTL 807 
The sequence alignments, and the active sites defined at distances of 4,5 or $6 \AA$ from the ligands, revealed a conserved sequence formed comprising one Leu residue (serving as gatekeeper) and the triplet Pro-Phe-Met (forming a hinge between the $\mathrm{N}$-terminal lobe and the $\mathrm{C}$-terminal lobe). The gatekeeper Leu regulates access to the hydrophobic specificity pocket, which comprises an Ala-Asp-Lys sequence followed by a single residue that differs with each TAM kinase (Ala in Tyro-3; Met in Axl; or Ile in Mer).

At $4 \AA$, the active site encompasses 45 amino acids, seven of which differ among the three TAM kinases. At $5 \AA$, the active site spans 59 amino acids, fifteen of which differ among the three kinases. Finally, at $6 \AA$, the active site is composed of 73 amino acids, nineteen of which differ by the TAM kinase. Analysis of the 3D-homology models of the three kinases revealed that, based on the orientations in the active site, there is only one key residue in both states, whose side chain is oriented inside the active site: Ala581 in Tyro-3; Met598 in Axl; and Ile650 in Mer (Figure 5). We reasoned that this amino acid must play a crucial role in the selectivity of ligands for each of the three TAM kinases. The 3D analysis also revealed that for all the active sites in each of the two conformations (DFG-Asp in and DFG-Asp out), the activation loop of the kinase domain formed part of the active site. This finding underscores the importance of using a model that includes the residues of the activation loop.

\subsection{Virtual Screening}

We sought to investigate the selectivity of ligands among the three TAM kinases, as well as the role of the key residue in each kinase (identified in the previous section), using virtual screening of known TAM inhibitors. Thus, we searched two databases (PubChem and CanSAR) and obtained a set of 156 compounds (Supplementary Table S1). Depending on the chemical structure, these ligands were docked into each member of the TAM family in either the DFG-Asp in (151 compounds) or the DFG-Asp out (five compounds) conformation.

Given the high number of ligands docked in the DFG-Asp in conformation models, we required a data-sorting step. We performed this step using a designed scoring function in an MS Excel (MS Office 2010) spreadsheet, which enabled us to ascertain the inhibition profile of each compound based on its IC50 values (Supplementary Table S1). This procedure afforded 29 ligands with a strong inhibition profile: five that are selective against Tyro-3; 22 that are selective against Mer; and two pan-inhibitors of the TAM kinases. By analyzing the docking results for these 29 compounds, and taking into consideration their $\mathrm{IC}_{50}$ values, we were able to determine the role of the key residue of each TAM kinase in ligand selectivity. The selected inhibitors were then divided into two chemical series based on their common structural cores: diaminopyrimidines and aminopyrazolopyrimidines.

\subsection{Insight into Selectivity}

\subsubsection{Diaminopyrimidine Scaffold}

For the first series, three inhibitors selective for Tyro-3 were docked into the Tyro-3 homology model. Analysis of the docking results revealed that all three inhibitors adopt the same position in the Tyro-3 active site as that seen in the crystallized structures of Mer containing inhibitors bearing the same structural core (PDB ID: 4MHA and 4MH7) [51]. For instance, with compound 104, three hydrogen bonds formed between the diaminopyrimidine moiety of the ligand and the backbones of three residues 
from the active site of the Tyro-3 model: the two hinge residues (Pro604 and Met606), and one residue in the roof of the ATP-binding site (Leu524) (Figure 6).

Figure 6. Comparison and overlapping of compound 104 docked in the Tyro-3 model aligned with the PDB ligands UNC1817 (PDB: 4MHA) and UNC1896 (PDB: 4MH7).

(a) Overview of the overlapping of the three ligands in the active site of the Tyro-3 model.

(b) Overview of the distance between the methoxyphenyl fragment in compound $\mathbf{1 0 4}$ and the Ala581 side chain of Tyro-3. (c) Overview of the distance between the methoxyphenyl fragment in compound 104 and either Met598 in Axl or the Ile650 side-chain in Mer.

(d) Representation of the three diaminopyrimidine-based inhibitors selective for Tyro-3.
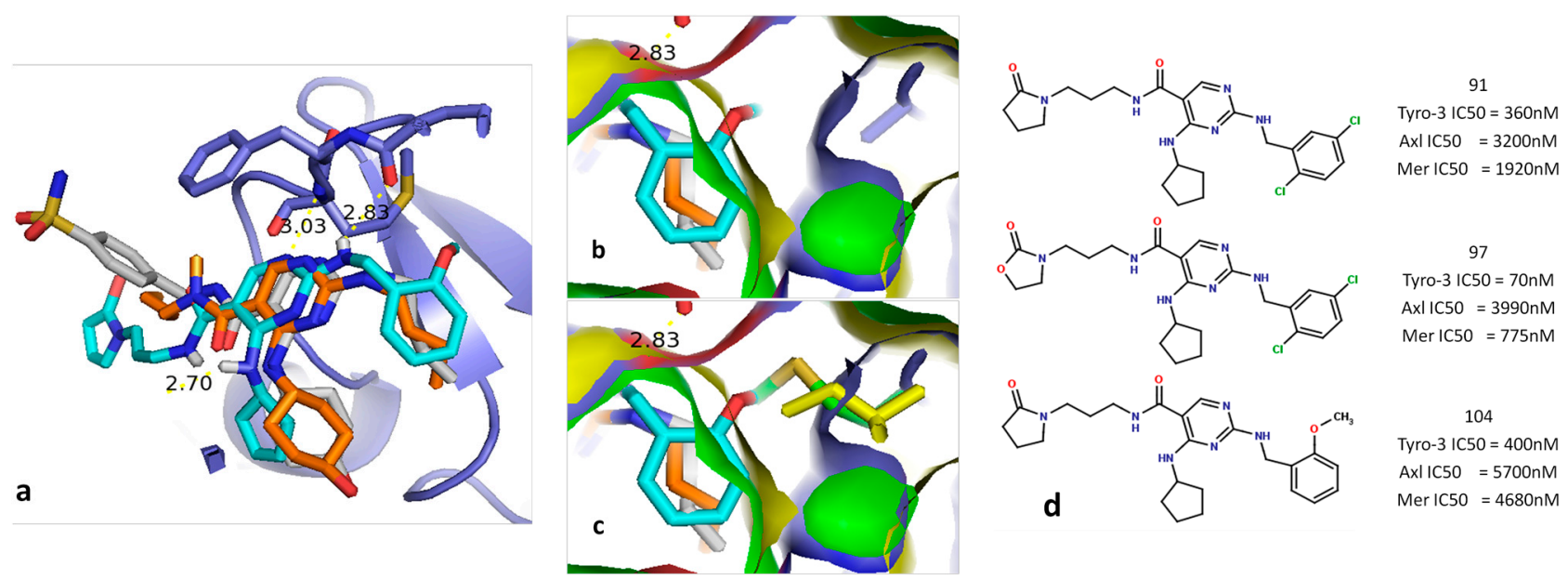

The study of the aforementioned docking conformation revealed that, as previously supposed by Zhang et al. [51], the overlapping key residues from each TAM kinase (Ala581 in Tyro-3; Met598 in Axl; and Ile650 in Mer) help determine the selectivity of a given ligand. As clearly shown in Figure 6, the methoxyphenyl group of compound 104 comes over Ile650 in Mer and Met598 in Axl (the methoxy fragment passes through the surface of Mer (in yellow) and of Axl (in green)), whereas it has much more space in Tyro-3, due to the small size of the Ala581 side chain. Similar steric constraints are observed for the 5-chloride group in compounds 91 and 97 when either ligand is docked inside the Tyro-3 model and subsequently overlapped with the Axl and Mer homology models. Hence, we reasoned that the key variable residue could be exploited in the design of selective, type I Tyro-3 inhibitors.

\subsubsection{Pyrazolopyrimidine Scaffold}

Our second series of ligands comprised 36 compounds designed around a pyrazolopyrimidine scaffold. Docking of these molecules in each of our three DFG-Asp in models (Tyro-3, Axl and Mer) confirmed the binding mode discovered by Liu et al. [49]: formation of two hydrogen bonds between the pyrazolopyrimidine scaffold and the hinge region (Pro672 and Met674 in Mer), and of two hydrogen bonds between the 4-aminocyclohexyl moiety and residues Arg727 and Asn728. All the active compounds in this series established these same four hydrogen bonds with the conserved amino acids of the three TAM kinase active sites. Thus, selectivity amongst the kinases can be explained only by the previously described differential key residue. For example, in compound 30, the methylamine substituent at position 
6 of the scaffold (Figure 7) can establish hydrophobic contacts with Ile650 in Mer or with Met598 in Axl, giving this inhibitor strong activities against these two kinases (IC50 Mer $=56 \mathrm{nM}$; and $\mathrm{IC}_{50} \mathrm{Axl}=81 \mathrm{nM}$ ).

Figure 7. Structure of compounds 16 (left) and $\mathbf{3 0}$ (right), and their respective activities against Tyro-3, Axl and Mer.
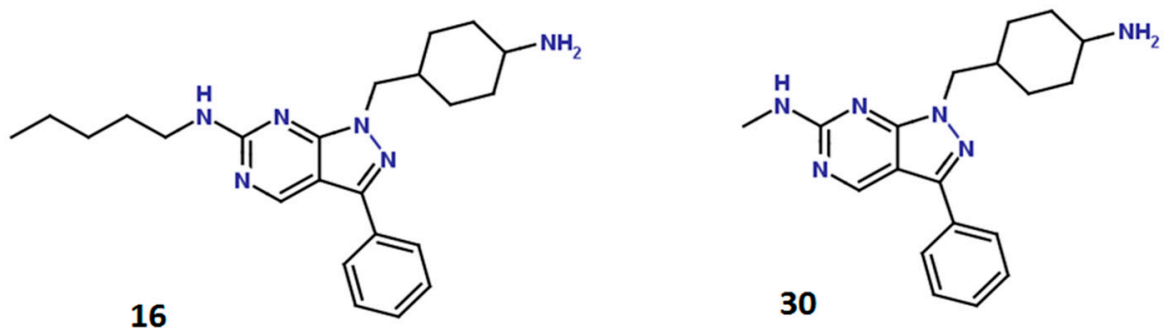

Tyro-3 IC50 $=77 \mathrm{nM}$
AxIIC50 $=47 \mathrm{nM}$
Mer IC50 $=14 \mathrm{nM}$

Tyro-3 IC50 = 1400nM

Axl IC50 $=81 \mathrm{nM}$

Mer IC50 $=56 \mathrm{nM}$

However, as the methylamine substituent is too short to interact with Ala583 in Tyro-3, the activity of compound 30 against this kinase is much weaker ( $\mathrm{IC}_{50}$ Tyro-3 $=1400 \mathrm{nM}$ ) (Figure 8). In contrast, compound 16, which features a pentylamine substituent, is very active against all three kinases (from highest to lowest activity: $\mathrm{IC}_{50} \mathrm{Mer}=14 \mathrm{nM} ; \mathrm{IC}_{50} \mathrm{Axl}=47 \mathrm{nM}$; and $\mathrm{IC}_{50}$ Tyro-3 $=77 \mathrm{nM}$ ). Interestingly, the pentylamine substituent of compound $\mathbf{1 6}$ is sufficiently long to enter the hydrophobic pocket in Tyro-3, where it interacts with the side-chain of Ala583 as well as other surrounding hydrophobic amino acids (Ala581, Pro604, Met606 and Ala672). This difference in surface size explains why type I inhibitors such as compound $\mathbf{3 0}$ are more selective for Axl and Mer than for Tyro-3.

Figure 8. Docking of compounds 30 and 16 in the Axl and Tyro-3 models. Representation of the docking of compound $\mathbf{3 0}$ in the active site of the Axl model (a) and of the Tyro-3 model (b). Interaction of the pentylamine substituent of compound $\mathbf{1 6}$ with Met-598 in Axl (c), or with the hydrophobic pocket created by Ala-581, Ala-583, Pro-604, Met-606 and Ala-672 in the active site of the Tyro-3 model (d).
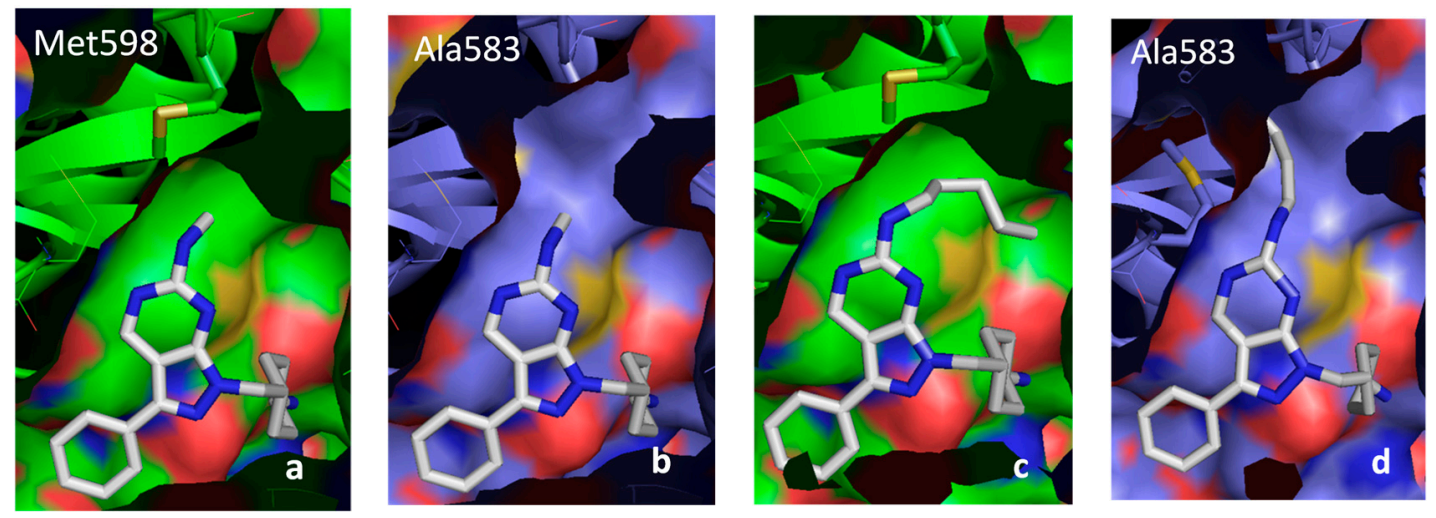

\subsubsection{DFG-Asp out Docking Analysis}

To analyze the DFG-Asp out conformations among the three TAM kinases, we collected five TAM DFG-Asp out inhibitors from online bioassay databases (Supplementary Table S2), and then docked 
each one in each of our DFG-Asp out models (Tyro-3, Axl and Mer). Upon close analysis of the docking of compounds 152-154 we identified the same orientation and binding mode in our models as those reported by Suarez et al. [54]. In this scenario, the purine core forms two hydrogen bonds with the hinge Met residue, the fluorophenyl group generates $\pi$-stacking interactions with the Phe of the DFG-Asp; the amide linker forms a hydrogen bond with the Asp of the DFG-Asp sequence, which also interacts with the carbonyl of the pyridine; and the terminal para-fluorophenyl moiety goes inside a hydrophobic pocket formed by Phe, Leu, and His in all three kinases (Figure 9). All of these interactions generate a potent type II TAM kinases inhibitor [54]. For the fluorine atom on the terminal phenyl moiety, the para position is that which leads to the highest activity; however, it generally corresponds to pan-TAM kinases inhibitors. Other highly potent TAM inhibitors such as compounds $\mathbf{1 5 2}$ and $\mathbf{1 5 4}$ bear the same general structure but differ in the heterocyclic group that binds to the hinge [46,47].

Figure 9. DFG-Asp out study. (a) Docking of compound 153 in the active site of the Mer DFG-Asp out model. (b) Structure of compounds 152-154 with their respective activities against Tyro-3, Axl and Mer. (c) General schematic of type II inhibitors docking in the active site of a TAM kinase in the DFG-Asp out conformation.

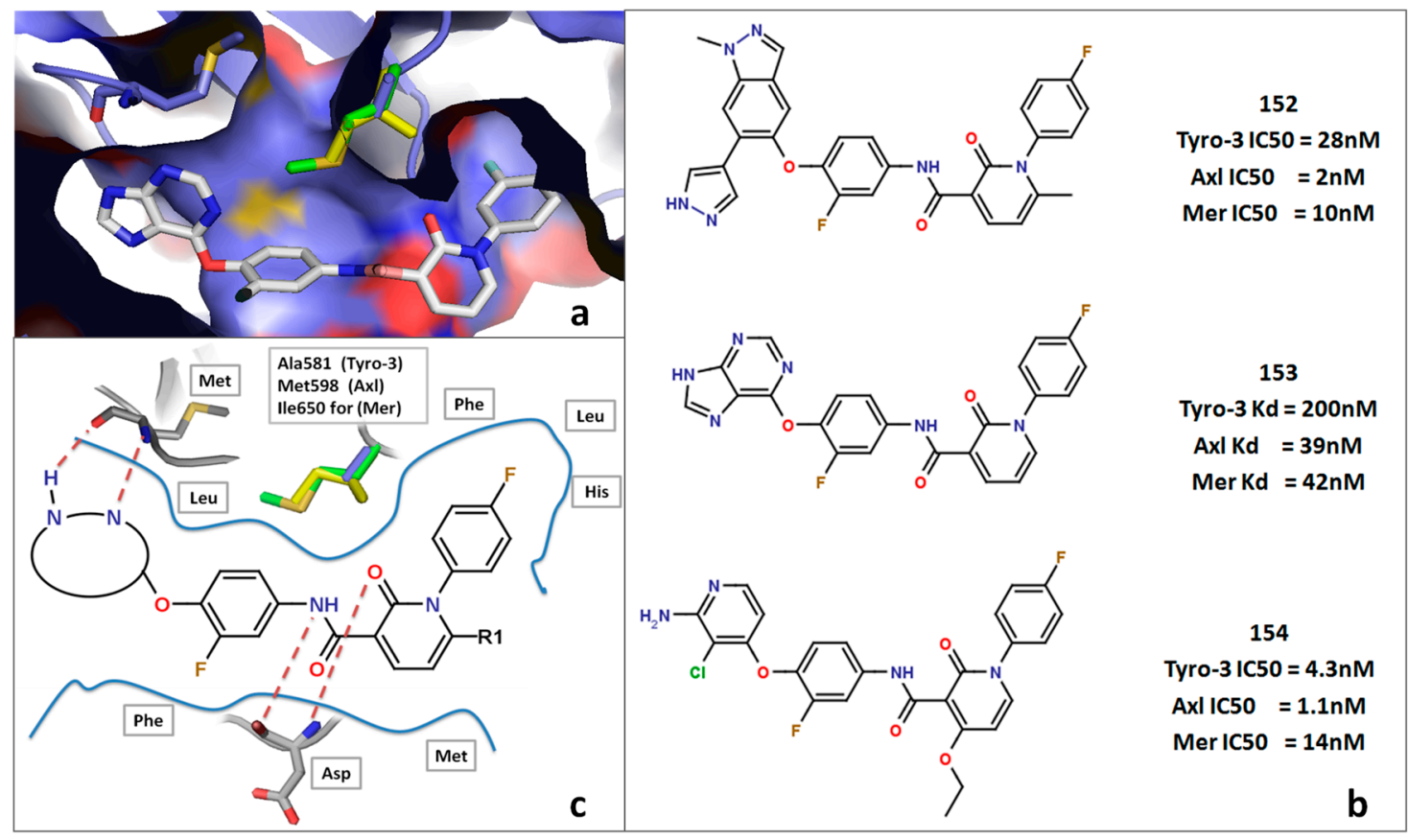

\section{Experimental Section}

\subsection{Data Sources}

There are 156 TAM inhibitors whose structures and $\mathrm{IC}_{50}$ values for each TAM enzyme are currently available; all of these data were retrieved from the CanSAR and PubChem databases [47,49-51,54-63]. The 3D structures of the template kinase c-Met in the DFG-Asp in and the DFG-Asp out conformations (2WD1 [52] and 3F82 [47], respectively) were retrieved from the Protein Data Bank [64]. The primary sequences of Tyro-3, Axl, Mer and c-Met were retrieved from the UniProt database (Uniprot ID: Q06418, P30530, Q12866, P08581 respectively) [65]. 


\subsection{Homology Modeling}

Sequences were aligned using CLUSTAL O (1.2.1) [66] multiple-sequence alignment from the Align tool of UniProt. The Swiss model server [67] was used to build the homology models of Tyro-3, Axl and Mer in each state (DFG-Asp in and DFG-Asp out). The templates used were the c-Met crystallized structures in either state. The obtained models were structurally validated using Procheck software [68]. Another validation was also performed using known inhibitors of TAM kinases.

\subsection{Docking}

We ran all 156 of the retrieved TAM inhibitors through virtual screening. Of these, 151 compounds docked in the three TAM kinase DFG-Asp in conformations, whereas five compounds docked in the corresponding DFG-Asp out conformations. Energy minimization was performed using Swiss Pdb-Viewer (v4.1.0) [69].

The docking files (ligand + protein active site) were prepared with Autodock Vina software [70], by using AutoDock Tools (v1.5.6) [71]. The following parameters were adjusted in this preparation step: (1) the Gastieger charges and polar hydrogens were added; (2) the grid-box dimensions were set at $20 \AA$ $(\mathrm{X}), 16 \AA(\mathrm{Y})$ and $24 \AA(\mathrm{Z})$ for the DFG-Asp in conformation, and $16 \AA(\mathrm{X}), 16 \AA(\mathrm{Y})$ and $28 \AA(\mathrm{Z})$ for the DFG-Asp out conformation; and (3) the center of the box was positioned at the midpoint of the active site, and the box volume covered the entire active site area plus a significant portion of the protein's solvent-exposed surface.

The following docking parameters were used in Autodock Vina: (1) all bonds in the inhibitor structures were allowed to rotate freely, except for the multiple bonds and the bonds in aromatic entities; (2) the kinase 3D structures were considered to be rigid; (3) a Lamarckian genetic algorithm was used for searching the conformational space in the active site; (4) the default grid spacing was set at $0.375 \AA$; (5) 100 different conformations were assessed, and the 20 highest scored binding modes were maintained for visual inspection; (6) the maximum energy difference between the best and the worst binding modes was set at $3 \mathrm{kcal} / \mathrm{mol}$; (7) the scoring function was a stochastic global optimization method inspired chiefly by X-Score [72]; and (8) visual inspection of the docking results, and image building, were done using PyMOL software [73].

\section{Conclusions}

The TAM receptor tyrosine kinases (Tyro-3, Axl and Mer) have been widely implicated in the development of cancer and other diseases; thus, they have become therapeutic targets of interest. In this study, we built homology models of each TAM kinase in each of its two states (DFG-Asp in and DFG-Asp out). We validated these models by two different methods, and then successfully overlapped them with existing partial 3D structures of Tyro-3 and of Mer. We highlighted the active site for each conformation and identified a single key residue that varies among the three kinases. We found a large set of type I TAM inhibitors in the literature, docked them in our three DFG-Asp in models, and subsequently sorted them according to their inhibition profiles, which paralleled the aforementioned key variable residue. This provided knowledge that could be exploited for the future SBDD of TAM inhibitors selective for Tyro-3 or for Mer/Axl. 
We also explored type II inhibitors, which bind to the TAM kinases in their DFG-Asp out conformations. As we found only a few inhibitors with defined activity against the TAM kinases, we did not have sufficient data with which to define a clear selectivity pattern. Moreover, we found that the key variable residue, which is paramount in the type I binding, is not very involved in the type II binding. However, we also determined that a terminal para-fluorophenyl group favors potent type II pan-TAM inhibitors. Further studies on additional type II inhibitors with defined TAM activities might enable a better understanding of the selectivity. We are presently using our findings and models from this study to design and synthesize new chemical entities as selective type I or type II TAM inhibitors.

\section{Supplementary Materials}

Supplementary materials can be accessed at: http://www.mdpi.com/1420-3049/19/10/16223/s1.

\section{Author Contributions}

A.M.: in charge of the docking studies and selectivity analysis; L.P.: in charge of the homology modeling, models validation of active sites determination; C.F.: wrote the Introduction, Material and Methods, and Conclusion, and managed the references; G.C.: principal investigator, and coordinator of the different studies; K.B.: principal investigator; A.Y.: principal investigator and corresponding author.

\section{Conflicts of Interest}

The authors declare no conflict of interest.

\section{References}

1. Manning, G.; Whyte, D.B.; Martinez, R.; Hunter, T.; Sudarsanam, S. The protein kinase complement of the human genome. Science 2002, 298, 1912-1934.

2. Varnum, B.C.; Young, C.; Elliott, G.; Garcia, A.; Bartley, T.D.; Fridell, Y.W.; Hunt, R.W.; Trail, G.; Clogston, C.; Toso, R.J. Axl receptor tyrosine kinase stimulated by the vitamin K-dependent protein encoded by growth-arrest-specific gene 6. Nature 1995, 373, 623-626.

3. Linger, R.M.A.; Keating, A.K.; Earp, H.S.; Graham, D.K. TAM receptor tyrosine kinases: Biologic functions, signaling, and potential therapeutic targeting in human cancer. Adv. Cancer Res. 2008, 100, 35-83.

4. Liu, E.; Hjelle, B.; Bishop, J.M. Transforming genes in chronic myelogenous leukemia. Proc. Natl. Acad. Sci. USA 1988, 85, 1952-1956.

5. O’Bryan, J.P.; Frye, R.A.; Cogswell, P.C.; Neubauer, A.; Kitch, B.; Prokop, C.; Espinosa, R., 3rd; Le Beau, M.M.; Earp, H.S.; Liu, E.T. Axl, a transforming gene isolated from primary human myeloid leukemia cells, encodes a novel receptor tyrosine kinase. Mol. Cell. Biol. 1991, 11, 5016-5031.

6. Neubauer, A.; Fiebeler, A.; Graham, D.K.; O’Bryan, J.P.; Schmidt, C.A.; Barckow, P.; Serke, S.; Siegert, W.; Snodgrass, H.R.; Huhn, D. Expression of axl, a transforming receptor tyrosine kinase, in normal and malignant hematopoiesis. Blood 1994, 84, 1931-1941. 
7. Graham, D.K.; Bowman, G.W.; Dawson, T.L.; Stanford, W.L.; Earp, H.S.; Snodgrass, H.R. Cloning and developmental expression analysis of the murine c-mer tyrosine kinase. Oncogene 1995, 10, 2349-2359.

8. Sun, W.S.; Fujimoto, J.; Tamaya, T. Coexpression of growth arrest-specific gene 6 and receptor tyrosine kinases Axl and Sky in human uterine endometrial cancers. Ann. Oncol. 2003, 14, 898-906.

9. Chung, B.I.; Malkowicz, S.B.; Nguyen, T.B.; Libertino, J.A.; McGarvey, T.W. Expression of the proto-oncogene Axl in renal cell carcinoma. DNA Cell Biol. 2003, 22, 533-540.

10. Gustafsson, A.; Boström, A.-K.; Ljungberg, B.; Axelson, H.; Dahlbäck, B. Gas6 and the receptor tyrosine kinase Axl in clear cell renal cell carcinoma. PLoS One 2009, 4, e7575.

11. Lin, W.C.; Li, A.F.; Chi, C.W.; Chung, W.W.; Huang, C.L.; Lui, W.Y.; Kung, H.J.; Wu, C.W. Tie-1 protein tyrosine kinase: A novel independent prognostic marker for gastric cancer. Clin. Cancer Res. 1999, 5, 1745-1751.

12. Wu, C.-W.; Li, A.F.Y.; Chi, C.-W.; Lai, C.-H.; Huang, C.L.; Lo, S.-S.; Lui, W.-Y.; Lin, W.-C. Clinical significance of AXL kinase family in gastric cancer. Anticancer Res. 2002, 22, 1071-1078.

13. Craven, R.J.; Xu, L.H.; Weiner, T.M.; Fridell, Y.W.; Dent, G.A.; Srivastava, S.; Varnum, B.; Liu, E.T.; Cance, W.G. Receptor tyrosine kinases expressed in metastatic colon cancer. Int. J. Cancer 1995, 60, 791-797.

14. Jacob, A.N.; Kalapurakal, J.; Davidson, W.R.; Kandpal, G.; Dunson, N.; Prashar, Y.; Kandpal, R.P. A receptor tyrosine kinase, $\mathrm{UFO} / \mathrm{Axl}$, and other genes isolated by a modified differential display PCR are overexpressed in metastatic prostatic carcinoma cell line DU145. Cancer Detect. Prev. 1999, 23, 325-332.

15. Mahajan, N.P.; Whang, Y.E.; Mohler, J.L.; Earp, H.S. Activated tyrosine kinase Ack1 promotes prostate tumorigenesis: Role of Ack1 in polyubiquitination of tumor suppressor Wwox. Cancer Res. 2005, 65, 10514-10523.

16. Sainaghi, P.P.; Castello, L.; Bergamasco, L.; Galletti, M.; Bellosta, P.; Avanzi, G.C. Gas6 induces proliferation in prostate carcinoma cell lines expressing the Axl receptor. J. Cell. Physiol. 2005, 204, 36-44.

17. Wu, Y.-M.; Robinson, D.R.; Kung, H.-J. Signal pathways in up-regulation of chemokines by tyrosine kinase MER/NYK in prostate cancer cells. Cancer Res. 2004, 64, 7311-7320.

18. Tanaka, K.; Nagayama, Y.; Nakano, T.; Takamura, N.; Namba, H.; Fukada, S.; Kuma, K.; Yamashita, S.; Niwa, M. Expression profile of receptor-type protein tyrosine kinase genes in the human thyroid. Endocrinology 1998, 139, 852-858.

19. Ito, T.; Ito, M.; Naito, S.; Ohtsuru, A.; Nagayama, Y.; Kanematsu, T.; Yamashita, S.; Sekine, I. Expression of the Axl receptor tyrosine kinase in human thyroid carcinoma. Thyroid 1999, 9, 563-567.

20. Ito, M.; Nakashima, M.; Nakayama, T.; Ohtsuru, A.; Nagayama, Y.; Takamura, N.; Demedchik, E.P.; Sekine, I.; Yamashita, S. Expression of receptor-type tyrosine kinase, Axl, and its ligand, Gas6, in pediatric thyroid carcinomas around chernobyl. Thyroid 2002, 12, 971-975.

21. Shieh, Y.-S.; Lai, C.-Y.; Kao, Y.-R.; Shiah, S.-G.; Chu, Y.-W.; Lee, H.-S.; Wu, C.-W. Expression of axl in lung adenocarcinoma and correlation with tumor progression. Neoplasia 2005, 7, 1058-1064.

22. Wimmel, A.; Glitz, D.; Kraus, A.; Roeder, J.; Schuermann, M. Axl receptor tyrosine kinase expression in human lung cancer cell lines correlates with cellular adhesion. Eur. J. Cancer 2001, $37,2264-2274$. 
23. Berclaz, G.; Altermatt, H.J.; Rohrbach, V.; Kieffer, I.; Dreher, E.; Andres, A.C. Estrogen dependent expression of the receptor tyrosine kinase axl in normal and malignant human breast. Ann. Oncol. 2001, 12, 819-824.

24. Tavazoie, S.F.; Alarcón, C.; Oskarsson, T.; Padua, D.; Wang, Q.; Bos, P.D.; Gerald, W.L.; Massagué, J. Endogenous human microRNAs that suppress breast cancer metastasis. Nature 2008, $451,147-152$.

25. Zantek, N.D.; Walker-Daniels, J.; Stewart, J.; Hansen, R.K.; Robinson, D.; Miao, H.; Wang, B.; Kung, H.J.; Bissell, M.J.; Kinch, M.S. MCF-10A-NeoST: a new cell system for studying cell-ECM and cell-cell interactions in breast cancer. Clin. Cancer 2001, 7, 3640-3648.

26. Meric, F.; Lee, W.-P.; Sahin, A.; Zhang, H.; Kung, H.-J.; Hung, M.-C. Expression profile of tyrosine kinases in breast cancer. Clin. Cancer Res. 2002, 8, 361-367.

27. Macleod, K.; Mullen, P.; Sewell, J.; Rabiasz, G.; Lawrie, S.; Miller, E.; Smyth, J.F.; Langdon, S.P. Altered ErbB receptor signaling and gene expression in cisplatin-resistant ovarian cancer. Cancer Res. 2005, 65, 6789-6800.

28. Sun, W.; Fujimoto, J.; Tamaya, T. Coexpression of Gas6/Axl in human ovarian cancers. Oncology 2004, 66, 450-457.

29. Tsou, A.P.; Wu, K.M.; Tsen, T.Y.; Chi, C.W.; Chiu, J.H.; Lui, W.Y.; Hu, C.P.; Chang, C.; Chou, C.K.; Tsai, S.F. Parallel hybridization analysis of multiple protein kinase genes: Identification of gene expression patterns characteristic of human hepatocellular carcinoma. Genomics 1998, 50, 331-340.

30. Vajkoczy, P.; Knyazev, P.; Kunkel, A.; Capelle, H.-H.; Behrndt, S.; von Tengg-Kobligk, H.; Kiessling, F.; Eichelsbacher, U.; Essig, M.; Read, T.-A.; et al. Dominant-negative inhibition of the Axl receptor tyrosine kinase suppresses brain tumor cell growth and invasion and prolongs survival. Proc. Natl. Acad. Sci. USA 2006, 103, 5799-5804.

31. Quong, R.Y.; Bickford, S.T.; Ing, Y.L.; Terman, B.; Herlyn, M.; Lassam, N.J. Protein kinases in normal and transformed melanocytes. Melanoma Res. 1994, 4, 313-319.

32. Györffy, B.; Lage, H. A Web-based data warehouse on gene expression in human malignant melanoma. J. Investig. Dermatol. 2007, 127, 394-399.

33. Van Ginkel, P.R.; Gee, R.L.; Shearer, R.L.; Subramanian, L.; Walker, T.M.; Albert, D.M.; Meisner, L.F.; Varnum, B.C.; Polans, A.S. Expression of the receptor tyrosine kinase Axl promotes ocular melanoma cell survival. Cancer Res. 2004, 64, 128-134.

34. Nakano, T.; Tani, M.; Ishibashi, Y.; Kimura, K.; Park, Y.-B.; Imaizumi, N.; Tsuda, H.; Aoyagi, K.; Sasaki, H.; Ohwada, S.; et al. Biological properties and gene expression associated with metastatic potential of human osteosarcoma. Clin. Exp. Metastasis 2003, 20, 665-674.

35. Crosier, P.S.; Hall, L.R.; Vitas, M.R.; Lewis, P.M.; Crosier, K.E. Identification of a novel receptor tyrosine kinase expressed in acute myeloid leukemic blasts. Leuk. Lymphoma 1995, 18, 443-449.

36. Challier, C.; Uphoff, C.C.; Janssen, J.W.; Drexler, H.G. Differential expression of the ufo/axl oncogene in human leukemia-lymphoma cell lines. Leukemia 1996, 10, 781-787.

37. De Vos, J.; Couderc, G.; Tarte, K.; Jourdan, M.; Requirand, G.; Delteil, M.C.; Rossi, J.F.; Mechti, N.; Klein, B. Identifying intercellular signaling genes expressed in malignant plasma cells by using complementary DNA arrays. Blood 2001, 98, 771-780. 
38. Zhang, Z.; Lee, J.C.; Lin, L.; Olivas, V.; Au, V.; LaFramboise, T.; Abdel-Rahman, M.; Wang, X.; Levine, A.D.; Rho, J.K.; et al. Activation of the AXL kinase causes resistance to EGFR-targeted therapy in lung cancer. Nat. Genet. 2012, 44, 852-860.

39. Mahadevan, D.; Cooke, L.; Riley, C.; Swart, R.; Simons, B.; Della Croce, K.; Wisner, L.; Iorio, M.; Shakalya, K.; Garewal, H.; et al. A novel tyrosine kinase switch is a mechanism of imatinib resistance in gastrointestinal stromal tumors. Oncogene 2007, 26, 3909-3919.

40. Liu, L.; Greger, J.; Shi, H.; Liu, Y.; Greshock, J.; Annan, R.; Halsey, W.; Sathe, G.M.; Martin, A.-M.; Gilmer, T.M. Novel mechanism of lapatinib resistance in HER2-positive breast tumor cells: Activation of AXL. Cancer Res. 2009, 69, 6871-6878.

41. Giles, K.M.; Kalinowski, F.C.; Candy, P.A.; Epis, M.R.; Zhang, P.M.; Redfern, A.D.; Stuart, L.M.; Goodall, G.J.; Leedman, P.J. Axl mediates acquired resistance of head and neck cancer cells to the epidermal growth factor receptor inhibitor erlotinib. Mol. Cancer Ther. 2013, 12, 2541-2558.

42. Song, X.; Wang, H.; Logsdon, C.D.; Rashid, A.; Fleming, J.B.; Abbruzzese, J.L.; Gomez, H.F.; Evans, D.B.; Wang, H. Overexpression of receptor tyrosine kinase Axl promotes tumor cell invasion and survival in pancreatic ductal adenocarcinoma. Cancer 2011, 117, 734-743.

43. Kaldor, S.W.; Kalish, V.J.; Davies, J.F., 2nd; Shetty, B.V.; Fritz, J.E.; Appelt, K.; Burgess, J.A.; Campanale, K.M.; Chirgadze, N.Y.; Clawson, D.K.; et al. Viracept (nelfinavir mesylate, AG1343): A potent, orally bioavailable inhibitor of HIV-1 protease. J. Med. Chem. 1997, 40, 3979-3985.

44. Wlodawer, A.; Vondrasek, J. Inhibitors of HIV-1 protease: A major success of structure-assisted drug design. Annu. Rev. Biophys. Biomol. Struct. 1998, 27, 249-284.

45. Kim, E.E.; Baker, C.T.; Dwyer, M.D.; Murcko, M.A.; Rao, B.G.; Tung, R.D.; Navia, M.A. Crystal structure of HIV-1 protease in complex with VX-478, a potent and orally bioavailable inhibitor of the enzyme. J. Am. Chem. Soc. 1995, 117, 1181-1182.

46. Feneyrolles, C.; Spenlinhauer, A.; Guiet, L.; Fauvel, B.; Daydé-Cazals, B.; Warnault, P.; Chevé, G.; Yasri, A. Axl kinase as a key target for oncology: Focus on small molecule inhibitors. Mol. Cancer Ther. 2014, 13, 2141-2148.

47. Schroeder, G.M.; An, Y.; Cai, Z.-W.; Chen, X.-T.; Clark, C.; Cornelius, L.A.M.; Dai, J.; Gullo-Brown, J.; Gupta, A.; Henley, B.; et al. Discovery of N-(4-(2-amino-3-chloropyridin-4-yloxy)-3fluorophenyl)-4-ethoxy-1-(4-fluorophenyl)-2-oxo-1,2-dihydropyridine-3-carboxamide (BMS-777607), a selective and orally efficacious inhibitor of the Met kinase superfamily. J. Med. Chem. 2009, 52, $1251-1254$.

48. Huang, X.; Finerty, P., Jr.; Walker, J.R.; Butler-Cole, C.; Vedadi, M.; Schapira, M.; Parker, S.A.; Turk, B.E.; Thompson, D.A.; Dhe-Paganon, S. Structural insights into the inhibited states of the Mer receptor tyrosine kinase. J. Struct. Biol. 2009, 165, 88-96.

49. Liu, J.; Yang, C.; Simpson, C.; Deryckere, D.; van Deusen, A.; Miley, M.J.; Kireev, D.; Norris-Drouin, J.; Sather, S.; Hunter, D.; et al. Discovery of novel small molecule Mer kinase inhibitors for the treatment of pediatric acute lymphoblastic leukemia. ACS Med. Chem. Lett. 2012, 3, 129-134.

50. Powell, N.A.; Hoffman, J.K.; Ciske, F.L.; Kaufman, M.D.; Kohrt, J.T.; Quin, J.; Sheehan, D.J.; Delaney, A.; Baxi, S.M.; Catana, C.; et al. Highly selective 2,4-diaminopyrimidine-5-carboxamide inhibitors of Sky kinase. Bioorg. Med. Chem. Lett. 2012, 23, 1046-1050. 
51. Zhang, W.; Zhang, D.; Stashko, M.A.; DeRyckere, D.; Hunter, D.; Kireev, D.; Miley, M.J.; Cummings, C.; Lee, M.; Norris-Drouin, J.; et al. Pseudo-cyclization through intramolecular hydrogen bond enables discovery of pyridine substituted pyrimidines as new Mer kinase inhibitors. J. Med. Chem. 2013, 56, 9683-9692.

52. Porter, J.; Lumb, S.; Franklin, R.J.; Gascon-Simorte, J.M.; Calmiano, M.; Riche, K.L.; Lallemand, B.; Keyaerts, J.; Edwards, H.; Maloney, A.; et al. Discovery of 4-azaindoles as novel inhibitors of c-Met kinase. Bioorg. Med. Chem. Lett. 2009, 19, 2780-2784.

53. Christoph, S.; Deryckere, D.; Schlegel, J.; Frazer, J.K.; Batchelor, L.A.; Trakhimets, A.Y.; Sather, S.; Hunter, D.M.; Cummings, C.T.; Liu, J.; et al. UNC569, a novel small-molecule mer inhibitor with efficacy against acute lymphoblastic leukemia in vitro and in vivo. Mol. Cancer Ther. 2013, 12, 2367-2377.

54. Suárez, R.M.; Chevot, F.; Cavagnino, A.; Saettel, N.; Radvanyi, F.; Piguel, S.; Bernard-Pierrot, I.; Stoven, V.; Legraverend, M. Inhibitors of the TAM subfamily of tyrosine kinases: Synthesis and biological evaluation. Eur. J. Med. Chem. 2013, 61, 2-25.

55. Bulusu, K.C.; Tym, J.E.; Coker, E.A.; Schierz, A.C.; Al-Lazikani, B. canSAR: Updated cancer research and drug discovery knowledgebase. Nucleic Acids Res. 2014, 42, D1040-D1047.

56. Wang, Y.; Suzek, T.; Zhang, J.; Wang, J.; He, S.; Cheng, T.; Shoemaker, B.A.; Gindulyte, A.; Bryant, S.H. PubChem BioAssay: 2014 Update. Nucleic Acids Res. 2014, 42, D1075-D1082.

57. Kitagawa, D.; Yokota, K.; Gouda, M.; Narumi, Y.; Ohmoto, H.; Nishiwaki, E.; Akita, K.; Kirii, Y. Activity-based kinase profiling of approved tyrosine kinase inhibitors. Genes Cells 2013, 18, 110-122.

58. Wang, X.-F.; Tian, X.-T.; Ohkoshi, E.; Qin, B.; Liu, Y.-N.; Wu, P.-C.; Hour, M.-J.; Hung, H.-Y.; Qian, K.; Huang, R.; et al. Design and synthesis of diarylamines and diarylethers as cytotoxic antitumor agents. Bioorg. Med. Chem. Lett. 2012, 22, 6224-6228.

59. Powell, N.A.; Kohrt, J.T.; Filipski, K.J.; Kaufman, M.; Sheehan, D.; Edmunds, J.E.; Delaney, A.; Wang, Y.; Bourbonais, F.; Lee, D.-Y.; et al. Novel and selective spiroindoline-based inhibitors of Sky kinase. Bioorg. Med. Chem. Lett. 2012, 22, 190-193.

60. Takeuchi, C.S.; Kim, B.G.; Blazey, C.M.; Ma, S.; Johnson, H.W.B.; Anand, N.K.; Arcalas, A.; Baik, T.G.; Buhr, C.A.; Cannoy, J.; et al. Discovery of a novel class of highly potent, selective, ATP-competitive, and orally bioavailable inhibitors of the mammalian target of rapamycin (mTOR). J. Med. Chem. 2013, 56, 2218-2234.

61. Zhang, D.; Ai, J.; Liang, Z.; Li, C.; Peng, X.; Ji, Y.; Jiang, H.; Geng, M.; Luo, C.; Liu, H. Discovery of novel 2-aminopyridine-3-carboxamides as c-Met kinase inhibitors. Bioorg. Med. Chem. 2012, 20, 5169-5180.

62. Zhang, W.; McIver, A.L.; Stashko, M.A.; DeRyckere, D.; Branchford, B.R.; Hunter, D.; Kireev, D.; Miley, M.J.; Norris-Drouin, J.; Stewart, W.M.; et al. Discovery of Mer specific tyrosine kinase inhibitors for the treatment and prevention of thrombosis. J. Med. Chem. 2013, 56, 9693-9700.

63. Yan, S.B.; Peek, V.L.; Ajamie, R.; Buchanan, S.G.; Graff, J.R.; Heidler, S.A.; Hui, Y.-H.; Huss, K.L.; Konicek, B.W.; Manro, J.R.; et al. LY2801653 is an orally bioavailable multi-kinase inhibitor with potent activity against MET, MST1R, and other oncoproteins, and displays anti-tumor activities in mouse xenograft models. Investig. New Drugs 2013, 31, 833-844.

64. Berman, H.M.; Westbrook, J.; Feng, Z.; Gilliland, G.; Bhat, T.N.; Weissig, H.; Shindyalov, I.N.; Bourne, P.E. The protein data bank. Nucleic Acids Res. 2000, 28, 235-242. 
65. Consortium, T.U. Activities at the universal protein resource (UniProt). Nucleic Acids Res. 2014, 42, D191-D198.

66. Sievers, F.; Wilm, A.; Dineen, D.; Gibson, T.J.; Karplus, K.; Li, W.; Lopez, R.; McWilliam, H.; Remmert, M.; Söding, J.; et al. Fast, scalable generation of high-quality protein multiple sequence alignments using Clustal Omega. Mol. Syst. Biol. 2011, 7, doi:10.1038/msb.2011.75.

67. Arnold, K.; Bordoli, L.; Kopp, J.; Schwede, T. The SWISS-MODEL workspace: a web-based environment for protein structure homology modelling. Bioinform. Oxf. Engl. 2006, 22, 195-201.

68. Laskowski, R.A.; MacArthur, M.W.; Moss, D.S.; Thornton, J.M. PROCHECK: A program to check the stereochemical quality of protein structures. J. Appl. Crystallogr. 1993, 26, 283-291.

69. Guex, N.; Peitsch, M.C. SWISS-MODEL and the Swiss-PdbViewer: An environment for comparative protein modeling. Electrophoresis 1997, 18, 2714-2723.

70. Trott, O.; Olson, A.J. AutoDock Vina: Improving the speed and accuracy of docking with a new scoring function, efficient optimization, and multithreading. J. Comput. Chem. 2010, 31, 455-461.

71. Goodsell, D.S.; Olson, A.J. Automated docking of substrates to proteins by simulated annealing. Proteins 1990, 8, 195-202.

72. Wang, R.; Lai, L.; Wang, S. Further development and validation of empirical scoring functions for structure-based binding affinity prediction. J. Comput. Aided Mol. Des. 2002, 16, 11-26.

73. The PyMol Molecular Graphics System, Version 0.99rc6; Schrodinger; Delano Scientific LLC: San Carlos, CA, USA, 2002.

Sample Availability: Not available.

(C) 2014 by the authors; licensee MDPI, Basel, Switzerland. This article is an open access article distributed under the terms and conditions of the Creative Commons Attribution license (http://creativecommons.org/licenses/by/4.0/). 